\title{
Observation of wave-packet propagation in the ion cyclotron range of frequencies in a tokamak plasma
}

\author{
G. J. Greene \\ Plasma Physics Laboratory, Princeton University, Princeton, New Jersey 08543 \\ R. W. Gould \\ California Institute of Technology, Pasadena, California 91125
}

(Received 13 December 1989; accepted 3 August 1990)

\begin{abstract}
Experimental observation of wave-packet propagation in the ion cyclotron range of frequencies in a tokamak plasma is reported. Studies were carried out in the Caltech Research Tokamak [Phys. Fluids 23, $614(1980)$ ] in a pure hydrogen plasma and in a regime where fast-wave damping was sufficiently small to permit multiple toroidal transits of the wave packet. Waves were launched by exciting a small loop antenna with a short burst of radio-frequency current and were detected with shielded magnetic probes. Probe scans revealed a large increase in wave-packet amplitude at smaller minor radii, and the packet velocity was found to be independent of radial position. Measurement of the packet transit time yielded direct information about the wave group velocity. Packet velocity was investigated as a function of the fundamental excitation frequency, plasma density, and toroidal magnetic field. Results are compared with the predictions of a cold plasma model that includes a vacuum layer at the edge.
\end{abstract}

\section{INTRODUCTION}

The study of propagation of plasma waves in the ion cyclotron range of frequencies (ICRF) is of particular interest because of their potential use for heating thermonuclear plasmas. In regimes where fast-wave damping is small, toroidal propagation of waves in a tokamak geometry leads to the formation of cavity resonances, or toroidal eigenmodes. These fast-wave modes were first observed in the TM-1-Vch and TO- 1 tokamaks, ${ }^{1,2}$ and have since been studied in a number of other devices. ${ }^{3-6}$

Toroidal eigenmodes are considered potentially attractive for plasma heating for two reasons. First, the resonant increase of electric field at the antenna causes an increase in the loading resistance of the wave-launching antenna. Since the loading resulting from excitation and damping of ICRF waves in tokamak plasmas is often small (on the order of a few ohms), an increase in antenna loading can improve the efficiency of power transfer to the plasma. Second, the eigenmodes are global modes, so energy can be deposited uniformly around the tokamak rather than in a localized region near the antenna.

A problem associated with the use of fast-wave eigenmodes for heating a reactor-sized plasma involves mode separation. For a fixed plasma density and toroidal magnetic field, there exists an infinite set of modes at discrete frequencies. The modes have finite width (in terms of frequency or density variation) as a result of wave damping, and the number of modes per unit frequency or density interval increases as the size of the tokamak and the plasma density increase. ${ }^{7}$ For fusion reactor regimes, there are expected to be a virtual continuum of overlapping modes. Simultaneous excitation of multiple modes can be undesirable as it leads to strong fields in the local vicinity of the antenna that may cause deleterious plasma edge heating. Nevertheless, there has becn continuing interest in utilizing the advantages of eigenmode plasma heating. ${ }^{8}$ Mode separation can be retained, for instance, by lowering the excitation frequency toward or below $\omega_{c i}$. Although the usual wave damping scenarios (utilizing second harmonic or minority fundamental resonances) would not be applicable, other mechanisms could perhaps lead to adequate absorption for plasma heating while retaining a sufficiently long damping length to permit eigenmode formation. For example, some studies have found anomalously high wave damping in singlc-species tokamak plasmas when the fundamental cyclotron resonance was in the plasma. ${ }^{5,9,10}$

Propagation of waves in the ICRF in tokamak plasmas has been the subject of considerable theoretical investigation, but experimental efforts in large tokamaks have been hampered by practical restrictions: limited accessibility to the plasma volume and the inability, in energetic plasmas, to utilize probes except in the outermost edge region. In smaller tokamaks with cooler plasmas, probes can be inserted to a significant fraction of the minor radius, and several investigations ${ }^{11,12}$ have used magnetic probes to examine the structure of the wave fields. Coincidently, the smaller size and lower plasma density associated with these devices allow operation in regimes where toroidal eigenmodes are distinct and well separated. Toroidal eigenmodes were observed in the Caltech Research Tokamak by Hwang and Gould ${ }^{13}$ as peaks on the antenna loading impedance, and wave measurements by $\mathrm{Greene}^{10}$ subsequently allowed mode identification. For the regime investigated $\left(\omega / \omega_{c i}=1-3, B_{0}=3-6\right.$ $\mathrm{kG}, \bar{n}_{c}=10^{12}-10^{13} \mathrm{~cm}^{-3}, I_{p}=15-25 \mathrm{kA}$, and $T_{e, i} \approx 50-$ $100 \mathrm{eV}$, hydrogen gas fill), calculations of relevant wave absorption mechanisms (first and second harmonic cyclotron damping, transit-time magnetic pumping (TTMP) and 
electron Landau damping, resistive wall damping, etc.) predicted toroidal wave damping lengths much longer than the tokamak circumference. Experimentally, high- $Q$ eigenmodes were, in fact, observed.

The wave response in the tokamak plasma regimes considered here is dominated by interference that leads, for fixed toroidal field and excitation frequency, to the formation of toroidal eigenmodes at the specific plasma densities where $k_{\|}=N / R$ ( $k_{\|}$is the toroidal wave number, $N$, an integer, is the toroidal mode number, and $R$ is the major radius). The large wave amplitude peaks observed as the plasma density sweeps through the resonances are very narrow and complicate wave studies, rendering some measurements useful only at the set of discrete eigendensities. It would be desirable for investigations of wave physics to be able to observe the propagation withoul interference effects, as if the waves were traveling in an infinite cylinder. Since the waves travel with finite toroidal group velocity, one possibility is to examine the propagation on a time scale short compared with the characteristic time for eigenmode formation, i.e., short compared with the transit time of the wave around the torus. Launching wave packets from an antenna with short bursts of $\mathrm{rf}$ current was found to be a useful approach to study wave propagation without interference effects. The time of flight of the wave disturbance around the tokamak yields direct information about the toroidal group velocity, and, in principle, the toroidal damping decrement can be determined as well.

Wave-packet techniques have been used previously in plasma physics, primarily to investigate the propagation of electron plasma surface waves in cylindrical geometry. ${ }^{14-16}$ A good review of this work was given by Moisan, Shivarova, and Trivelpiece. ${ }^{17}$ In tokamaks, the only previous use of this approach appears to have been an experiment that launched lower hybrid waves in the Doublet IIa tokamak. ${ }^{18}$

In this paper we describe wave-packet propagation experiments performed in the Caltech Research Tokamak. The experimental apparatus and method are described in Sec. II. ICRF wave packets were successfully launched and detected in a regime where fast-wave damping was small. Multiple toroidal transits of the packet around the vessel were observed, and characterization of the transit time allowed study of the wave group velocity as a function of various parameters. These experimental results are presented in Sec. III, along with data from radial probe scans which confirmed that the packet was a global plasma phenomena rather than a mode existing only near the plasma surface. In Sec. IV we describe a simple cold-plasma model that was used for numerical calculations of the expected group velocity, and comparison with the experimental data is discussed in Sec. $V$. Conclusions and a suggestion for a future application are presented in Sec. VI.

\section{EXPERIMENTAL METHOD}

The experiments reported here were carried out in the Caltech Research Tokamak, a small device of circular cross section with an air-core Ohmic heating transformer. The major and minor radii were 45.7 and $16.2 \mathrm{~cm}$, respectively, and there was no separate limiter. The tokamak operated with toroidal magnetic fields of 3-6 kG, plasma currents of $15-35 \mathrm{kA}$, and plasma densities of $10^{12}-10^{13} \mathrm{~cm}^{-3}$. Discharge lengths were typically $10-20 \mathrm{msec}$, and ion and electron temperatures were $50-100 \mathrm{eV}$. The machine was operated solely with hydrogen gas, and lengthy pulse-discharge cleaning was employed before each experimental run. The loop voltage, plasma current, and line-averaged electron density for a typical discharge are shown in Fig. 1. Note that gas puffing was used after the initial breakdown phase to provide a nearly linear density ramp. This allowed observation of successive eigenmodes during experiments with continuous rf excitation. A detailed description of this machine and its diagnostics and rf systems is contained in Ref. 10 .

The antenna used to excite waves for these experiments was a small loop $(7.3 \mathrm{~cm}$ in poloidal extent), which was encased in a narrow ceramic box. The diameter of the copper rod forming the loop was $0.63 \mathrm{~cm}$, and the toroidal extent of the shielding box was $1.9 \mathrm{~cm}$. The antenna was installed in a port on the horizontal midplane on the low-field side of the machine. In operation, the antenna was inserted $2.5 \mathrm{~cm}$ past the inner wall of the tokamak (with the normal to the plane of the loop oriented in the toroidal direction) and at this position no change in gross plasma behavior was observed. The antenna loop was connected to a $1 \mathrm{~m}$ long coaxial feeder tube that provided the vacuum seal and connection to the if source.

A diagram of the wave-packet propagation experiment is shown in Fig. 2. At a frequency of $12 \mathrm{MHz}$, the duration of a typical rf burst was approximately $350 \mathrm{nsec}$. To generate
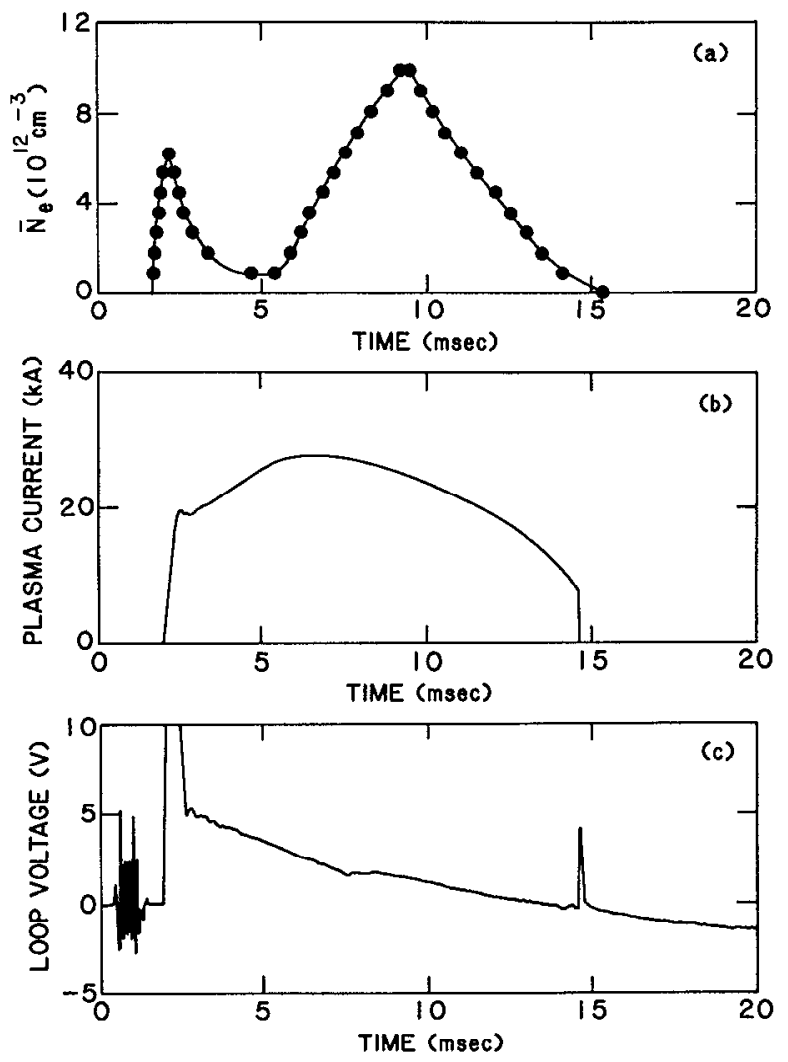

FIG. 1. Plasma parameters for a typical tokamak discharge. (a) Line-averaged electron density (from a microwave interferometer). (b) Plasma current. (c) One-turn loop voltage. 


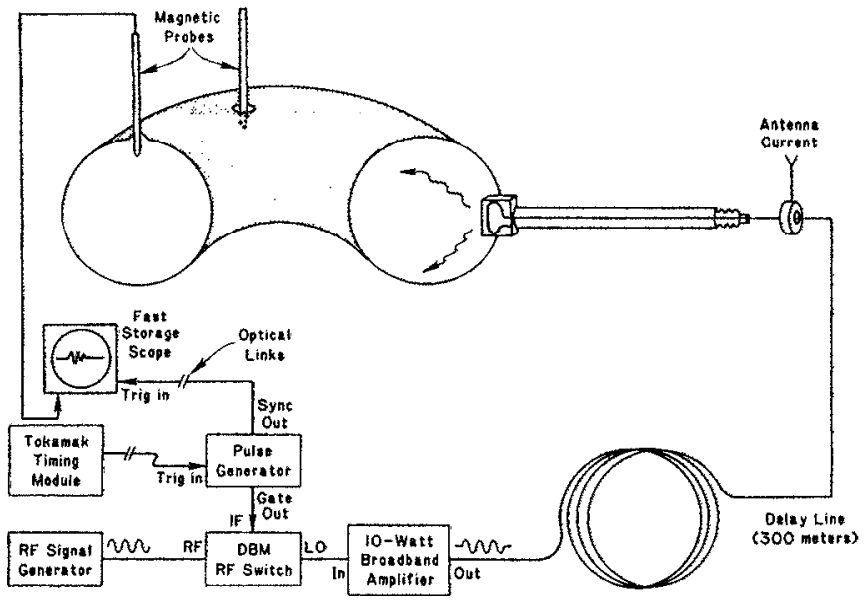

FIG. 2. Diagram of the wave-packet propagation experiment.

this waveform, a double-balanced mixer was used as a fast $\mathrm{rf}$ switch to gate the output of a continuous signal generator. The output of the mixer was amplified with a broadband 10 $\mathrm{W}$ amplifier and was directly connected, via $50 \Omega$ coaxial cable, to the antenna. Since the impedance mismatch between the cable and the antenna was very large (an impedance-matching network was not used in order to prevent unwanted ringing of the antenna current), the rf pulse incident on the antenna gave rise to a significant reflected pulse. This reflected pulse traversed the cable back to the generator, at which point another reflection occurred, sending a pulse back again toward the antenna. The object of this experiment was to excite the rf antenna with a short, isolated burst of current. Therefore the cable between the generator and the antenna was made sufficiently long $(300 \mathrm{~m})$ that the round-trip propagation time for the reflected wave $(\sim 3$ $\mu \mathrm{sec}$ ) was long compared to the time scales of interest. In order to prevent excessive power loss in the incident pulse, the delay line was made from a large-diameter cable.

The antenna current waveform was observed with a broadband rf current monitor mounted coaxially at the end of the antenna feeder tube. The wave fields in the tokamak were detected with an electrostatically shielded wideband if magnetic probe that responded to the time derivative of the toroidal component of the field. This probe consisted of a small coil ( $3 \mathrm{~mm}$ diam), coated with a lacquer of controlled conductivity (to provide an electrostatic shield while not perturbing the rf fields) and inserted in a glass vacuum envelope. The overall diameter of the probe envelope was $1 \mathrm{~cm}$ and it could be inserted radially at least $5 \mathrm{~cm}$ past the vacuum vessel wall without disturbing the gross characteristics of the plasma discharge.

The only available data recording system capable of responding in the submicrosecond time scales of these experiments was a $100 \mathrm{MHz}$ bandwidth storage oscilloscope. Scope traces were recorded with a standard camera. In order to simplify operation, the cables leading from the magnetic probe and from the antenna current monitor to the oscilloscope were matched in length. The inherent time delays as- sociated with the current monitor and the rf probe were investigated and found to be negligible.

In operation, a tokamak timing module provided the signal to start the wave-packet experiment. The timing signal, which could be delayed to any portion of the tokamak discharge, triggered a pulse generator with two outputs. One output with a variable width was used to gate the rf switch; the other triggered the fast oscilloscope. Since only one highspeed storage channel was available, it had to be switched between the excitation signal (the rf antenna current) and the received signal (from the magnetic probe) on a discharge-by-discharge basis. In order to ascertain the propagation time between the launched wave and the received signal, it was important that the oscilloscope trigger at precisely the same time from one discharge to the next. Timing pulses were carried by high-speed optical links, as shown in Fig. 2, and triggering jitter was found to be negligible.

The observation of wave-packet propagation in this experiment required proper design of the excitation current waveform. It was desirable to make the toroidal extent of the wave packet smaller than the major circumference of the vessel in order to maximize the resolution of the time-delay measurement (i.e., to prevent the leading edge of the wave packet from overlapping with its own trailing edge). The spatial length of the wave packet could be shortened by reducing the time duration of the current excitation pulse. This did not necessarily lead to an increase in clarity of the observed signals, however, because shortening of the pulse width in time resulted in a broadening of its frequency spectrum. As a result of nonlinear dispersion $\left(\partial^{2} \omega / \partial k^{2} \neq 0\right)$, the envelope of a wave packet spreads out as it travels, and the shorter the excitation pulse, the greater the broadening. Experimentally, the optimum antenna current pulse typically consisted of 3-5 cycles. Since wave packets were presumed to propagate symmetrically from the antenna in both toroidal directions (asymmetry resulting from the static poloidal magnetic field was expected to be small), the magnetic probe was located diametrically opposite the vessel from the antenna. That position optimized the received signal envelope separation as it allowed the greatest time interval between successive passes of the two oppositely traveling wave packets.

\section{EXPERIMENTAL RESULTS}

Raw probe and current-monitor signals for a typical experiment are shown in Figs. 3(a) and 3(b). The probe was located in a vertical port $180^{\circ}$ toroidally and $90^{\circ}$ poloidally away from the $r f$ antenna, the fundamental excitation frequency $(f)$ was $12 \mathrm{MHz}$, and the toroidal magnetic field on axis $\left(B_{0}\right)$ was $4.0 \mathrm{kG}$ (hence $\omega / \omega_{c i} \approx 2$ on the tokamak axis). The rf pulse was triggered at a time during the plasma discharge when the line-averaged density $\left(\bar{n}_{e}\right)$ was $8 \times 10^{12}$ $\mathrm{cm}^{-3}$. The antenna current pulse shown in the upper trace of Fig. 3(a) is about four periods long. The received probe signal consists of a series of approximately equally spaced bursts of oscillations with distinct envelopes of diminishing peak amplitude, and the first burst is delayed with respect to the antenna current. These signals are interpreted as result- 

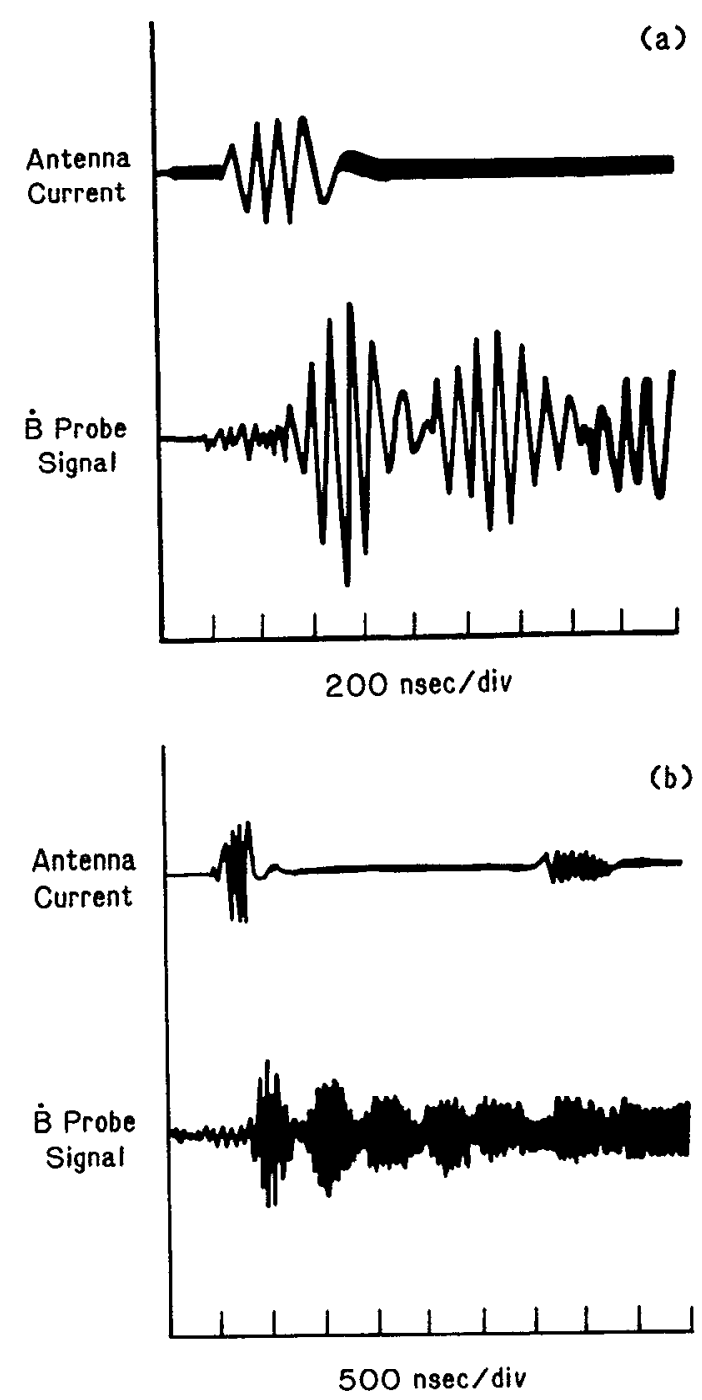

FIG. 3. Raw antenna current and magnetic probe signals for two typical tokamak discharges recorded at different sweep rates. Here $f=12 \mathrm{MHz}$, $B_{0}=4.0 \mathrm{kG}$, and $\bar{n}_{c}=8 \times 10^{12} \mathrm{~cm}^{-3}$. (a) A $2 \mu \mathrm{sec}$ sweep period. (b) A 5 $\mu$ sec sweep period.

ing from wave packets traveling symmetrically in opposite directions around the torus. The envelope of the first received burst occurs when the packets have traveled halfway around the major circumference of the tokamak; the second envelope results from the packets traveling another full circumference. Note that the time delay between the approximate centers of the antenna current envelope and the first received signal envelope is roughly half the time delay between the centers of the first and second received signal envelopes. This observation is consistent with the idea that the disturbance travels torodially with constant velocity.

The traces of Fig. 3(b) show the raw signals from another tokamak discharge on a longer time scale; experimental parameters were approximately the same as above. The small signal appearing $3 \mu \mathrm{sec}$ after the first burst on the antenna current trace is the first reflection from the incident pulse (having traveled through the delay line to the amplifier and back to the antenna again). The received probe signal displays at least four distinct envelopes of diminishing am- plitude. The successive envelopes appear to become broader and eventually the signal loses its coherent structure. The envelopes that are distinct are approximately equally spaced in time, supporting the hypothesis that they result from wave packets making multiple transits around the tokamak.

\section{A. Parameter scans}

Approximately 500 tokamak discharges were recorded while changing various plasma and if parameters. The resulting data set was analyzed to yield the packet velocity as a function of the rf fundamental excitation frequency, the toroidal magnetic field, and the plasma density. The packet velocity was defined as the major toroidal circumference $(2.87 \mathrm{~m})$ divided by the time delay between the centers of the first and second probe signal envelopes. Because the signal records were photographic in nature, the digital data analysis techniques routinely used for other diagnostics were not available. Tracings were made of the signals on each photograph and the envelopes were approximated; measurements were then made under a microscope with a scale. This procedure was subject to some arbitrary judgments. Nevertheless, reproducibility of the experimental data and subsequent analysis was adequate $( \pm 10 \%-20 \%)$ to clearly establish useful trends in the behavior of the wave-packet velocity. Typically, results from 3-5 plasma discharges were averaged for each experimental data point shown in Figs. 4-8.

The dependence of packet velocity on plasma centralchord-averaged density is shown by the experimental points in Fig. 4 (the solid lines are results of calculations from the model discussed later). The excitation frequency was 12 $\mathrm{MHz}$ and the toroidal field on axis was $4.0 \mathrm{kG}$. The density during the experiment was increased by keeping the discharge and gas puff constant and varying the time during the discharge at which the rf pulse was triggered. The results

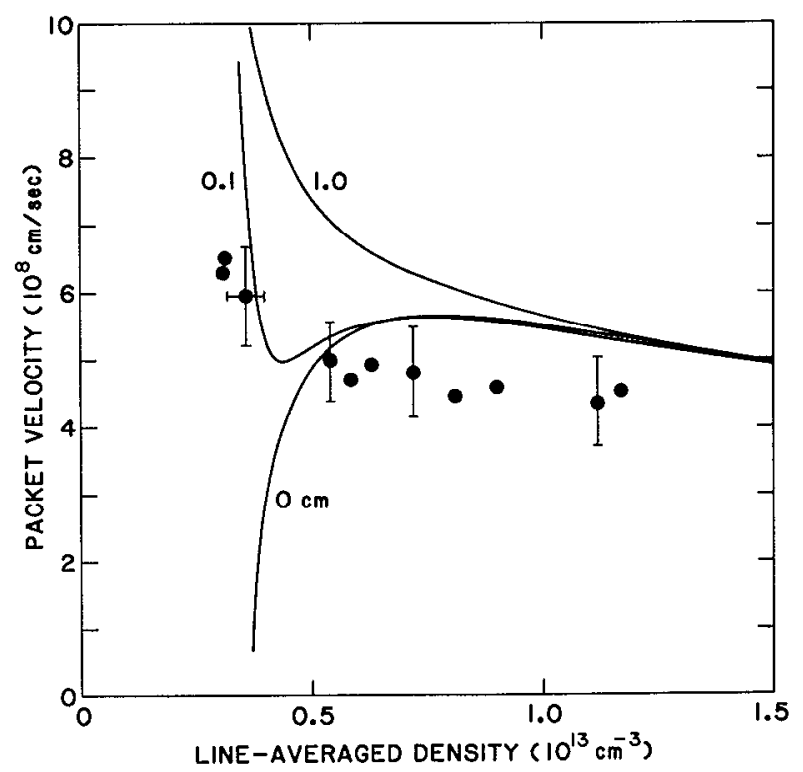

FIG. 4. Variation of the wave-packet velocity with line-averaged electron density. The points are experimental data, while the three solid lines represent calculations from the theoretical model using values for the vacuum layer thickness as indicated. For these data $f=12 \mathrm{MHz}$ and $B_{0}=4.0 \mathrm{kG}$. 


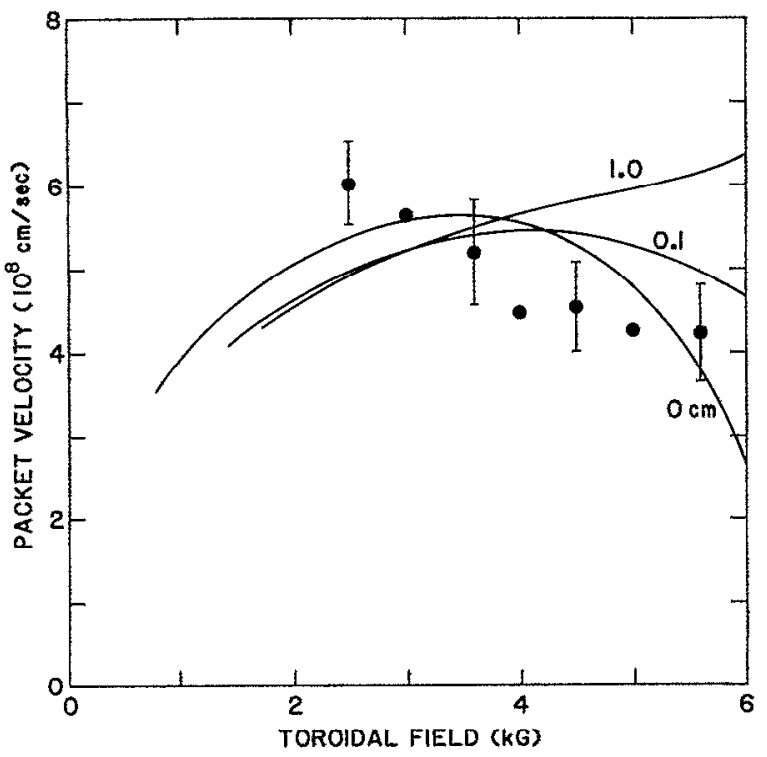

FIG. 5. Variation of the wave-packet velocity with the value of the static toroidal magnetic field on the tokamak axis. The points and solid lines are as described in Fig. 4. Here $f=12 \mathrm{MHz}$ and $\bar{n}_{t^{\prime}}=8 \times 10^{12} \mathrm{~cm}^{-3}$.

show that the velocity decreased by some $30 \%$ as the density increased from $3 \times 10^{12}$ to $1.2 \times 10^{13} \mathrm{~cm}^{-3}$.

Variation of the packet velocity with toroidal magnetic field is shown in Fig. 5. Here the excitation frequency was 12 $\mathrm{MHz}$ and the plasma density at the time of the experiment was $8 \times 10^{12} \mathrm{~cm}^{-3}$. The velocity decreased somewhat as the toroidal field increased, changing by approximately $30 \%$ as the field varied from $2.5-5.6 \mathrm{kG}$.

The variation of wave-packet velocity with the rf excita-

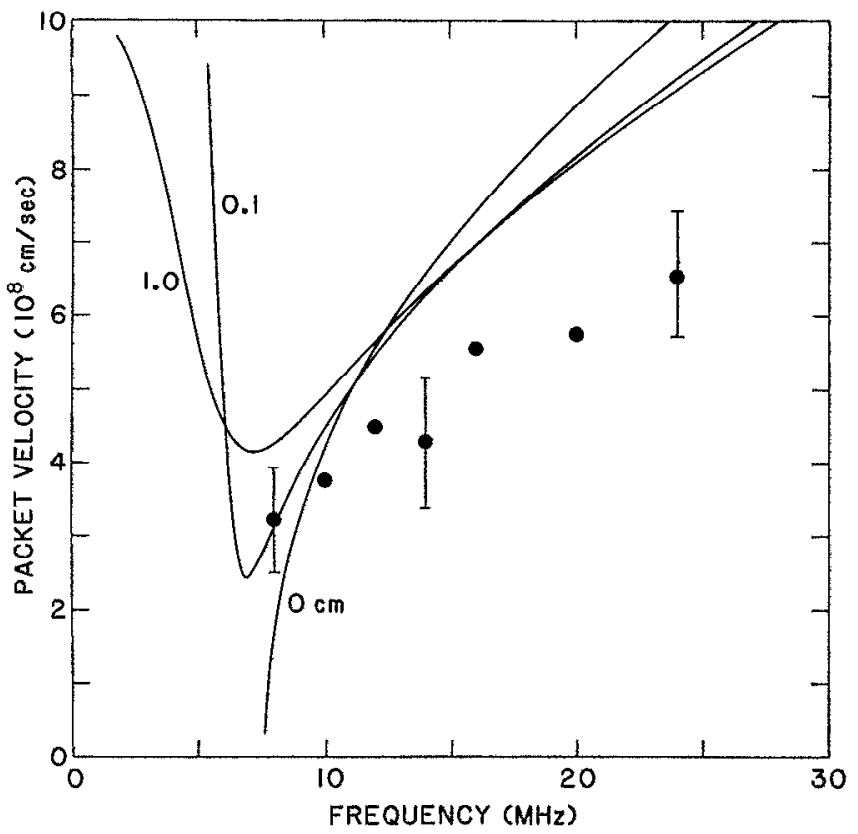

FIG. 6. Variation of the wave-packet velocity with excitation frequency. The points and solid lines are as described in Fig. 4. Here $\bar{n}_{10}=8 \times 10^{12}$ $\mathrm{cm}^{-3}$ and $B_{0}=4.0 \mathrm{kG}$.

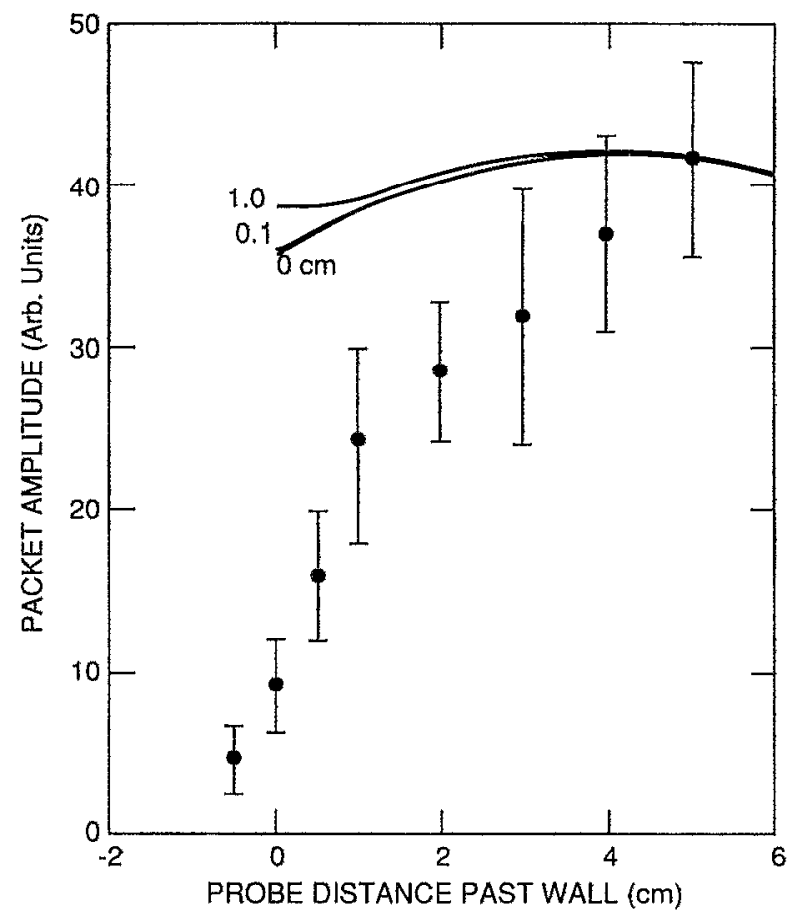

FIG. 7. Radial dependence of the wave-packet amplitude detected by a magnetic probe located $180^{\circ}$ toroidally and $90^{\circ}$ poloidally away from the if antenna. The wall is located at a minor radius of $16.2 \mathrm{~cm}$. For these data, $f=12 \mathrm{MHz}, B_{t \mathrm{f}}=4.0 \mathrm{kG}$, and $\bar{n}_{t .}=1.0 \times 10^{13} \mathrm{~cm}^{-3}$. The points represent experimental data, and the solid lines are the results of the theoretical model with the thickness of the vacuum layer as indicated and the amplitude normalized to match the experimental value at the innermost probe location.

tion frequency is shown by the experimental points in Fig. 6. The toroidal magnetic field on axis was $4.0 \mathrm{kG}$ and the lineaveraged density was $8 \times 10^{12} \mathrm{~cm}^{-3}$. The velocity increased by a factor of about 2 over a range of frequency from 8-24 MHz.

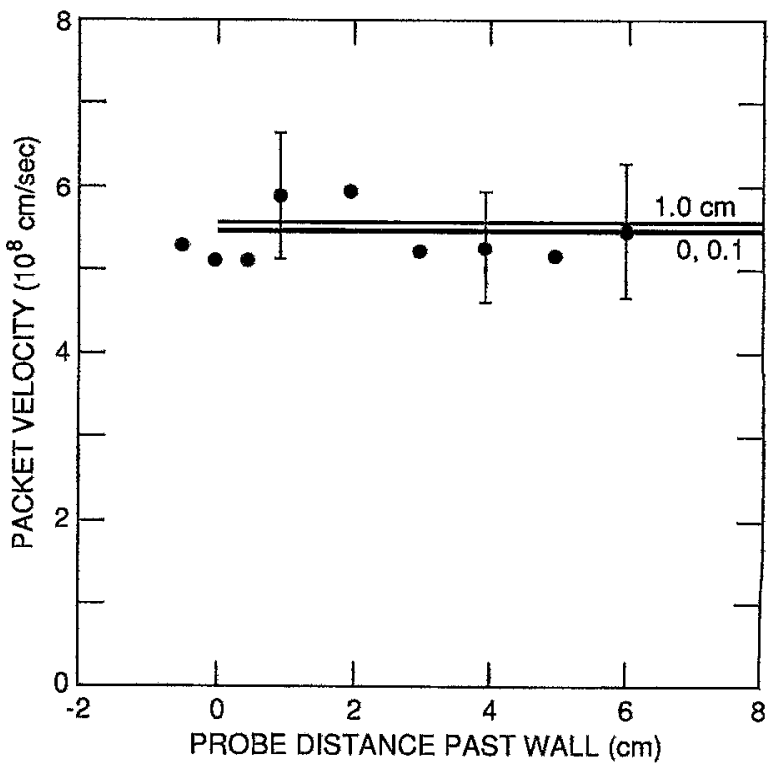

FIG. 8. Radial dependence of the wave-packet velocity. Conditions were the same as in Fig. 7. The points and solid lines are as described in Fig. 4 , 


\section{B. Probe radial scans}

Experiments were performed to investigate the radial dependence of the packet amplitude and velocity of propagation.

The peak amplitude of the first burst on the probe signal is plotted as a function of the probe position in Fig. 7. The excitation frequency for this experiment was $12 \mathrm{MHz}$, the toroidal field on axis was $4.0 \mathrm{kG}$, and the plasma density at the time of the peak of the rf pulse was $1 \times 10^{13} \mathrm{~cm}^{-3}$. The packet amplitude increased substantially as the probe moved into the plasma, varying by a factor of 10 over the range of positions investigated.

Variation of the wave-packet velocity with the radial position of the probe is shown in Fig. 8; the experimental conditions were the same as for Fig. 7. There was no significant change in the packet velocity as the probe moved up to 6 $\mathrm{cm}$ into the plasma.

\section{THEORETICAL MODEL}

A disturbance localized in space can be represented as

$$
B(\mathbf{x}, t)=\int A(\mathbf{k}) e^{i(\mathbf{k} \cdot \mathbf{x} \cdot \omega t)} d^{3} \mathbf{k},
$$

where $B$ is some field component and $\mathbf{k}$ is the wave vector, related to the angular frequency $\omega$ through a dispersion relation. For a localized wave packet, the coefficient $A(\mathrm{k})$ is peaked around some specific wave number $\mathbf{k}_{c}$. If the dispersion is not too great, then expansion of $\omega$ about $\mathbf{k}_{c}$ leads to

$$
B(\mathbf{x}, t)=B\left(\mathbf{x}-\frac{\partial \omega}{\partial \mathbf{k}} t, 0\right) \exp \left[i\left(\left.\mathbf{k}_{c} \cdot \frac{\partial \omega}{\partial \mathbf{k}}\right|_{\mathbf{k}_{c}}-\omega_{c} t\right)\right],
$$

where $\omega_{c}=\omega\left(\mathbf{k}_{c}\right)$, and the envelope of the initial disturbance progagates without change in form at the group velocity $\mathbf{v}_{g}=\partial \omega / \partial \mathbf{k} .^{19}$ Including higher-order terms in the expansion shows that the form of the packet spreads out or broadens as it propagates. In this analysis, the exact mechanism of excitation of the initial disturbance is not considered (treatment of the complete initial value problem leads, for instance, to the phenomena of forerunners ${ }^{20}$ ). Motivated by the experimental results, we seek the toroidal group velocity of an existing wave-packet disturbance, and the appropriate dispersion relation $\omega(\mathbf{k})$ must be found.

To describe the plasma wave propagation, we consider a uniform, cold, cylindrical plasma of radius $r_{1}$ and infinite length concentric with a perfectly conducting cylindrical shell of radius $r_{2}$. A vacuum layer exists between the plasma and the conducting boundary. The axis of the cylinder is taken to be the $z$ axis of the coordinate system, the azimuthal coordinate is $\theta$, and the radial coordinate $r$. The background magnetic field $B_{0}$ is assumed to be uniform and directed along the cylinder axis. The $z$ component of the wave electric field in the plasma is neglected (equivalent to the approximation of neglecting electron mass) and solutions to Maxwell's equations of the form $e^{i\left(k_{1} z+m \theta-\omega t\right)}$ are sought where $k_{\|}$is the parallel wave number and $m$, an integer, is the azimuthal mode number. Following Allis, Buchsbaum, and Bers, ${ }^{2}$ for $r<r_{1}$ the result is simply

$$
B_{z}(r)=J_{m}(p r) \text {, }
$$

where the field is normalized so that $B_{z}\left(r_{1}\right)=J_{m}\left(p r_{1}\right)$ and $p$, the radial wave number, is given by

$$
p^{2}=\frac{k_{0}^{4}\left(\epsilon_{1}^{2}-\epsilon_{x}^{2}\right)+k_{\|}^{4}-2 k_{0}^{2} k_{\|}^{2} \epsilon_{1}}{k_{0}^{2} \epsilon_{1}-k_{\|}^{2}} .
$$

Here $k_{0}=\omega / c$, where $c$ is the speed of light, and the elements related to the cold plasma dielectric tensor are

$$
\begin{aligned}
& \epsilon_{\perp}-1-\sum_{i} \frac{\omega_{p i}^{2}}{\omega^{2}-\omega_{c i}^{2}}, \\
& \epsilon_{\times}=\sum_{i} \frac{\omega_{p i}^{2} s_{i}}{\omega^{2}-\omega_{c i}^{2}} \frac{\omega_{c i}}{\omega},
\end{aligned}
$$

where $\omega_{c i}$ and $\omega_{p i}$ are the cyclotron and plasma frequencies for species $i, s_{i}$ is the sign of the charge of species $i$, and the summation is over species (electron and protons in this case).

In the vacuum region, $r_{1}<r<r_{2}$, solutions of the wave equations are

$$
\begin{aligned}
& B_{z}(r)=\alpha I_{m}(T r)+\beta K_{m}(T r), \\
& E_{z}(r)=\gamma I_{m}(T r)+\delta K_{m}(T r),
\end{aligned}
$$

where $\alpha, \beta, \gamma$, and $\delta$ are constants, $I_{m}$ and $K_{m}$ are the modified Bessel functions, and $T^{2}=k^{2}-k_{0}^{2}$.

Boundary conditions at the plasma-vacuum interface require continuity of the tangential electric field and the $z$ component of the magnetic field. ${ }^{22}$ At the vacuum-conducting shell interface, the tangential electric field and normal magnetic field must vanish. Note that the $\theta$ component of the magnetic field will not, in general, be continuous across the plasma-vacuum interface because the infinite plasma conductivity in the $z$ direction arising from neglect of the electron mass permits a surface current to flow in that direction.

Continuity of $E_{z}$ at $r=r_{1}$, together with the condition $E_{z}\left(r_{2}\right)=0$, requires that $E_{z}\left(r_{1}\right)=0$; hence $E_{z}$ vanishes everywhere. The remaining field components in both regions can be written in terms of $B_{z}$ and $\partial B_{z} / \partial r$. In particular, for $r<r_{1}$, we find

$$
E_{\theta}(r)=i \frac{m}{r} F_{1} B_{z}(r)+i F_{2} \frac{\partial B_{z}(r)}{\partial r},
$$

where $\quad F_{1}=k_{0}^{3} \epsilon_{\times} / D, \quad F_{2}=\left(k_{0} k_{\|}^{2}-k_{0}^{3} \epsilon_{1}\right) / D, \quad$ and $D=k_{\|}^{4}-2 k_{0}^{2} k_{\|}^{2} \epsilon_{1}+k_{0}^{4}\left(\epsilon_{1}^{2}-\epsilon_{\times}^{2}\right)$. In the vacuum region, $r_{1}<r<r_{2}$, it is easily shown that

$$
E_{\theta}(r)=i \frac{k_{0}}{T^{2}} \frac{\partial B_{z}(r)}{\partial r}
$$

and

$$
B_{r}=-i \frac{k_{\|}}{T^{2}} \frac{\partial B_{z}(r)}{\partial r} .
$$

The boundary condition $B_{r}\left(r_{2}\right)=0$ then yields a relation between $\alpha$ and $\beta$ :

$$
\frac{\alpha}{\beta}=\frac{r_{2} T K_{m+1}\left(T r_{2}\right)-m K_{m}\left(T r_{2}\right)}{r_{2} T I_{m+1}\left(T r_{2}\right)+m I_{m}\left(T r_{2}\right)} .
$$

Continuity of the $z$ component of the magnetic field at the plasma-vacuum interface gives another relation between $\alpha$ and $\beta$ : 


$$
\beta=\left[J_{m}\left(p r_{1}\right)-\alpha I_{m}\left(T r_{1}\right)\right] / K_{m}\left(T r_{1}\right)
$$

Equations (1) and (2) allow solution for $\alpha$ and $\beta$ in terms of the plasma parameters. Continuity of the $\theta$ component of the electric field at the plasma-vacuum interface then yields the following relation:

$$
\begin{gathered}
\frac{m}{r_{1}} F_{1} J_{m}\left(p r_{1}\right)+F_{2}\left(\frac{m}{r_{1}} J_{m}\left(p r_{1}\right)-p J_{m+1}\left(p r_{1}\right)\right) \\
=\frac{k_{0}}{T^{2}}\left[\alpha\left(T I_{m+1}\left(T r_{1}\right)+\frac{m}{r_{1}} I_{m}\left(T r_{1}\right)\right)\right. \\
\left.+\beta\left(\frac{m}{r_{1}} K_{m}\left(T r_{1}\right)-T K_{m+1}\left(T r_{1}\right)\right)\right] .
\end{gathered}
$$

Since $\alpha$ and $\beta$ are already determined, Eq. (3) represents the wave dispersion relation, connecting $\omega$ to $k_{\|}$, the plasma parameters, and the geometry.

For sufficiently high density or frequency, or sufficiently low magnetic field, Eq. (3) may have more than one real solution for $k_{\|}$, corresponding to different radial modes. We refer to these solutions by the index $l$, where $l=1$, the first radial mode, is the solution that has the largest value of $k_{\}}$. Previous work ${ }^{10}$ has shown, for the case with no vacuum layer, that over much of the range of parameters considered in these experiments, the only toroidally propagating mode is the $m=1, l=1$ mode. The exact boundaries of parameters for which only one mode is propagating depend on the exact density profile, which is, in general, not known. The experiments described here showed distinct wave packets propagating over a wide range of parameters with a radial amplitude dependence that appeared monotonic. The propagation of more than one mode would lead to a distortion of the packet envelope and a change in radial amplitude profile. We assume for these calculations that only the $m=1, l=1$ mode is excited, permitting straightforward evaluation of a unique toroidal group velocity $\partial \omega / \partial k_{\|}$.

Inclusion of the electron mass terms in the wave equations introduces a second value of radial wave number $p$ for each toroidal wave number $k_{\|}$. For the parameters appropriate to the experiments considered here, this value of $p$ is generally complex, resulting in solutions of the form $I_{m}(|p| r)$. These solutions are significant only near the plasma boundary; neglect of this branch of the wave equation is experimentally justified since there was no evidence of radial wave field distributions peaked at the surface.

\section{DISCUSSION}

The theoretical calculations for the dependence of wavepacket group velocity on density, frequency, and toroidal magnetic field are shown by the solid lines in Figs. 4-6. In each case, three curves are shown corresponding to calculations with a vacuum layer $1.0 \mathrm{~cm}$ thick $\left(r_{1}=15.2 \mathrm{~cm}\right)$, a layer $0.1 \mathrm{~cm}$ thick $\left(r_{1}=16.1 \mathrm{~cm}\right)$, and no vacuum layer $\left(r_{1}=16.2 \mathrm{~cm}\right)$.

The behavior of the group velocity as a function of the plasma density (averaged along a central chord to correspond to the actual measurement of the microwave interfer- ometer) is shown in Fig. 4. A marked difference is observed between the form of the group velocity with and without a vacuum layer. In the absence of a vacuum layer, the group velocity decreases as the density falls for $\bar{n}_{e} \leqslant 7 \times 10^{12} \mathrm{~cm}^{-3}$; for a layer $1 \mathrm{~cm}$ thick, the velocity monotonically increases as the density decreases within the range shown.

For a uniform plasma that completely fills the conduct-. ing shell, it can be shown that the propagating modes all suffer a cutoff $\left(v_{\text {phase }} \rightarrow \infty, v_{\text {group }} \rightarrow 0\right)$ at a particular density and that the wave does not propagate below that density. It was first shown by Paoloni ${ }^{23}$ that inclusion of a vacuum layer between the plasma and the conducting boundary eliminates the cutoff and allows waves to propagate to very low densities. The dramatic difference in the behavior of the group velocity near the cutoff density appropriate to the case with no vacuum layer is a manifestation of this effect. The experimental data points in this region agree reasonably well with the calculations that use a $0.1 \mathrm{~cm}$ thick vacuum layer.

Calculations showing the dependence of the group velocity on the toroidal field, for fixed excitation frequency and plasma density, are plotted in Fig. 5. Here the largest deviations between the three calculations occur at high fields, where the case with a $1 \mathrm{~cm}$ vacuum layer predicts a signifcantly higher group velocity than was actually observed.

Finally, the variation of group velocity as a function of excitation frequency is plotted for the three cases in Fig. 6 . The largest differences between the calculations occur at low frequency, where the case without a vacuum layer shows a cutoff while the other two cases do not. The best fit at low frequency is obtained from the calculation with a $0.1 \mathrm{~cm}$ thick vacuum layer, but all three cases show significant deviation from experimental points at higher frequencies (this could result from a breakdown of the assumption of single mode propagation). The increase in group velocity occurring at frequencies below that corresponding to cutoff for the calculation without a vacuum layer could not be confirmed as data points below $8 \mathrm{MHz}$ were not available.

Although significant approximations were made in the theoretical model (neglect of toroidicity, magnetic field gradient, poloidal field, electron mass and thermal effects, assumption of a single propagating mode and a constant density profile with a sharp boundary, etc.), the trends in group velocity predicted for an assumed vacuum layer $0.1 \mathrm{~cm}$ thick are in moderate agreement with the experimental wavepacket velocity data. It should be noted that the lack of a limiter in the Caltech Research Tokamak means that the region at the plasma edge where the density is very low (i.e., sufficiently low that $\omega>\omega_{\text {lower hybrid }}$ ) is quite thin. Langmuir probe studies suggest that this layer is, in fact, much less than $1 \mathrm{~cm}$ thick.

The radial probe scans of amplitude and velocity (Figs. 7 and 8) demonstrate that the wave packet is not simply a surface phenomenon: the fields extend throughout the plasma cross section and the packet propagates uniformly in the toroidal direction, as expected from the results of the model. There is good agreement between the predicted and observed magnitude of the packet velocity. The radial dependence of the wave-packet amplitude, however, is one area of disagreement between the experimental data and the model. 
The observed field falls off faster at the edge than the variation predicted by the model $\left[J_{1}(p r)\right]$ in the absence of a vacuum layer. The model predicts an even smaller decrease in the field amplitude at the edge when a vacuum layer is included. The same discrepancy between the experimental data and the model was seen previously ${ }^{10}$ for $B_{z}$ field profiles at eigenmode resonances during experiments with continuous excitation. In that case, independent measurements confirmed that the poloidal dependence of the fields was appropriate to the $m=1$ mode. It is possible that the difference between the experimental results and the theoretical predictions for field profiles near the edge may arise from the actual boundary conditions at the wall, which differ from the assumptions of the model because the probe is introduced through a $2.2 \mathrm{~cm}$ diam port in the wall of the vessel. The tangential conducting surface that supports $B_{z}$ at the edge by allowing surface currents to flow in the poloidal direction is thus absent from the local vicinity of the probe.

\section{CONCLUSIONS}

Wave packets in the ion cyclotron range of frequencies have been successfully launched and detected in a tokamak plasma. The amplitude of the wave magnetic field associated with the packet increased toward smaller minor radii while the velocity of propagation was approximately constant, indicating that the packet traveled as a global plasma disturbance and not as a surface phenomenon. Several traverses of the packet around the tokamak circumference could be observed before packet broadening led to loss of coherence. The group velocity was obtained from the transit time of the wave packet and was investigated as a function of plasma density, excitation frequency, and static toroidal magnetic field. The magnitudes of the observed velocities ranged from one to three times the Alfvén speed. The packet velocity decreased with increasing density or toroidal magnetic field and rose with increasing excitation frequency. Trends in the experimental results were compared with the predictions of a simple cold-plasma model, which considered a single propagating mode, and reasonable quantitative and qualitative agreement was found when a thin vacuum layer was included at the plasma edge.

In a different form, the wave-packet technique for investigating fundamental physical properties of ICRF waves might prove useful in larger and hotter tokamak plasmas. Although the toroidal damping lengths in machines such as TFTR and JET may be too small to observe toroidal propagation, the dimensions and densities are such that it might be feasible to launch wave packets that would propagate radially. It is speculated that this approach could yield a method of probing the supposed radial reflections that occur at various resonance layers in these plasmas.

\section{ACKNOWLEDGMENT}

This work was supported by the U.S. Department of Energy under Contracts No. DE-AS03-76-SFO-0767 and No. DE-ACO2-76-CHO-3073.

'N. V. Ivanov, I. A. Kovan, and E. V. Los, JETP Lett. 14, 138 (1971). ${ }^{2}$ V. L. Vdovin, O. A. Zinov'ev, A. A. Ivanov, L. L. Kozorovitskii, V. V. Parail, Ya. R. Rakhimbabaev, and V. D. Rusanov, JETP Lett. 14, 149 (1971).

${ }^{3}$ J. Adam, M. Chance, H. Eubank, W. Getty, E. Hinnov, W. Hooke, J. Hosea, F. Jobes, F. Perkins, R. Sinclair, J. Sperling, and H. Takahashi, in Plasma Physics and Controlled Nuclear Fusion Research 1974, Proceedings of the 5th International Conference, Tokyo, (IAEA, Vienna, 1975), Vol. 1, pp. 65-73.

${ }^{4}$ See National Technical Information Service Document No. PPPL-1545 (H. Takahashi, Princeton Plasma Physics Laboratory Report No. PPPL$1545,1979)$. Copies may be ordered from the National Technical Information Service, Springfield, Virginia 22161 . The price is $\$ 17.00$ plus a $\$ 3.00$ handling fee. All orders must be prepaid.

${ }^{5}$ TFR Group, in Plasma Physics and Controlled Nuclear Fusion Research 1976, Proceedings of the 6th International Conference, Berchtesgaden, (IAEA, Vienna, 1977), Vol. III, pp. 39-59.

${ }^{\circ} \mathrm{H}$. R. Thompson, Ph.D. thesis, Princeton University, 1984.

${ }^{7}$ T. H. Stix, Nucl. Fusion 15, 737 (1975).

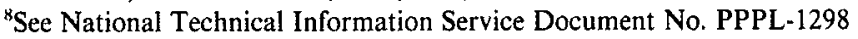
(T. II. Stix, Princeton Plasma Physics Laboratory Report No. PPPL1298, 1976). Copies may be ordered from the National Technical Information Service, Springfield, Virginia 22161 . The price is $\$ 11.00$ plus a $\$ 3.00$ handling fee. All orders must be prepaid.

${ }^{9}$ V. P. Bhatnagar, G. Bosia, M. Bures, E. Desoppere, A. M. Messiaen, C. Piret, G. Telesca, P. E. Vandenplas, and R. R. Weyants, in Proceedings of the Joint Varenna-Grenoble International Symposium on Heating in Toroidal Plasmas, Grenoble, 1978 (Pergamon, Oxford, 1979), Vol. 1, pp. 133-138.

${ }^{10} \mathrm{G}$. J. Greene, Ph.D. thesis, California Institute of Technology, 1984.

'R. J. Taylor and G. J. Morales, in Proceedings of the 3rd Topical Conference on Radio Frequency Heating (California Institute of Technology, Pasadena, 1978), pp. C5-1-C5-3.

${ }^{12}$ P. D. Coleman, Ph.D. thesis, Texas Technical University, 1983.

${ }^{13}$ D. Q. Hwang and R. W. Gould, Phys. Fluids 23, 614 (1980).

${ }^{14}$ Q. Demokan, H. C. S. Hsuan, and K. E. Lonngren, Plasma Phys. 13, 29 (1971).

${ }^{15}$ B. A. Anicin, V. M. Babovic, and K. E. Lonngren, J. Plasma Phys. 7, 403 (1972).

${ }^{16}$ D. L. Landt, H. C. S. Hsuan, and K. E. Lonngren, Plasma Phys. 16, 407 (1974).

${ }^{17}$ M. Moisan, A. Shivarova, and A. W. Trivelpiece, Plasma Phys. 24, 1331 (1982).

${ }^{18}$ See National Technical Information Service Document No. DE90016206 (J. L. Luxon et al, General Atomic Company Report No. GAA15820, 1980). Copies may be ordered from the National Technical Information Service, Springfield, Virginia 22161 . The price is $\$ 17.00$ plus a $\$ 3.00$ handling fee. All orders must be prepaid.

${ }^{19} \mathrm{~J}$. D. Jackson, Classical Electrodynamics (Wiley, New York, 1962), p. 210.

${ }^{20} \mathrm{~L}$. Brillouin, Wave Propagation and Group Velocity (Academic, New York, 1960).

${ }^{2}$ W. P. Allis, S. J. Buchsbaum, and A. Bers, Waves in Anisotropic Plasmas (M.I.T. Press, Cambridge, 1963).

${ }^{22}$ T. H. Stix, The Theory of Plasma Waves (McGraw-Hill, New York, 1962), p. 86.

${ }^{23}$ F. J. Paoloni, Phys. Fluids 18, 640 (1975). 